\title{
Electron Impact Ionization Mass Spectra of 3-Substituted-2-hydroxy-4(3H)-quinazolinones
}

\author{
1. M. El-Deen ${ }^{\wedge}$ and M. E. Abd El-Fattah ${ }^{\dot{*}}$ \\ Faculty of Lducation, Sue Canal University, Port-Said, Egyt \\ 'Faculty of Science, Sue Canal Uninersity, Ismailia, Egyt \\ Received August 9, 2002
}

\begin{abstract}
3-Amino-2-hydroxy-4(3H)-quinazolinone (3) was prepared via condensation of 1 with hydrazine hydrate. Treatment of 3 with appropriate acid in $\mathrm{POCl}_{3}$, ethyl chloroacetate and activated olefinic compounds in DMF yielded the corresponding 3-(substituted)amino-2-hydroxy-4(3H)-quinazolinones 4,5 and $\mathbf{6}$. The electron impact ionization mass spectra of compounds 3 and $\mathbf{4}$ show a weak molecular ion peak and a base peak of $m / z$ I 46 resulting from a cleavage fragmentation. The compounds 5 and 6 give a characteristic fragmentation pattern with a very stable fragment of benzopyrazolone $(\mathrm{m} / \mathrm{s} 132)$.
\end{abstract}

Key Words : Mass spectroscopy, 3-Substituted-2-lydroxy-quinazolinones

\section{Introduction}

In the course of recent investigations ${ }^{1-8}$ involving anthranilic acid and phenyl isocyanate, it was found that $2-(3-$ phenylureido)-benzoic acid (1) is converted into 3-amino-2hydroxy-4 $(3 \mathrm{H})$-quinazolinone $(3)$ by the action of hydrazine hydrate under fussion. The fact that only limited information is available on the mass spectra of 3-amino-2-hydroxy$4(3 \mathrm{H})$-quinazolinone $(3)$, along with the preparation of a novel 3-substituted-2-hydroxy-4(3H)-quinazolinone, has prompted us to report their synthesis and study their electron impact (El) mass spectral fragmentation.

\section{Results and Discussion}

Chemistry. Hydrazinolysis of 2-(3-phenylureido)benzoic acid (1) with hydrazine hydrate by fusion at $150^{\circ} \mathrm{C}$, gave the corresponding 3-amino-2-hydroxy-4(3H)-quinazolinone (3). which does not give the expected structure 2 (Scheme 1). Compound 3 may be formed by the nucleophilic attack at carbonyl groups in compound $\mathbf{1}$ with ring cyclization via the removal of water and aniline as shown in Scheme 2 .

the 3 -(alkylcarbonyl)amino-2-hydroxy-4(3H)-quinazolinones (4a-c) were synthesized by a acylation or benzoylation of the 3-amino-2-hydroxy-4(3H)-quinazolinone (3) with appropriate acid (such as acetic acid, chloroacetic acid, and benzoic acid) in phosphours oxychloride.

Alkylation of compound $\mathbf{3}$ with ethyl chloroacetate in dimethyl formamide under reflux formed the ethyl 2hydroxy-4(3H)-oxo-quinazoline-3-ylaminoacetic ester (5, Scheme 3). The reaction of 3-amino-2-hydroxy-4(3H)quinazolinone (3) with activated olefinic compound (such as acrylonitrile and methyl acrylate) in dimethyl formamide resulted in the formation of 3-(cyanoethyl)amino-2-hydroxy$4(3 \mathrm{H})$-quinazolinone $(6 \mathrm{a})$ and ethyl 2 -hydroxy-4(3H)-oxoquinazolin-3-yl-3-aminopropionic ester (6b, Scheme 2), respectively.

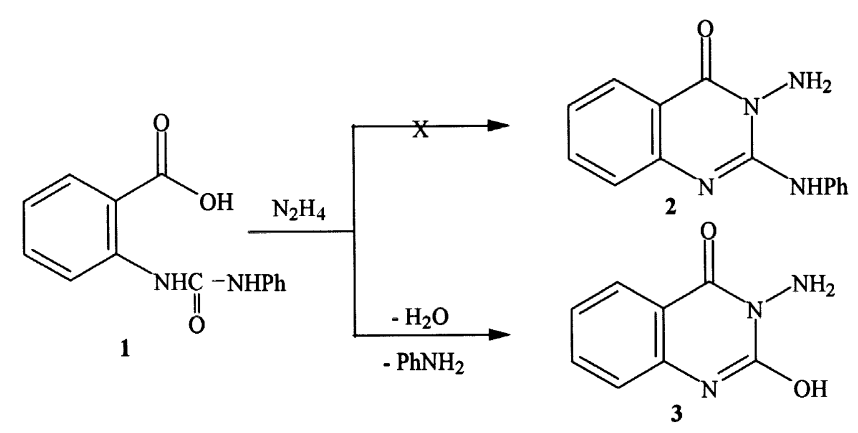

Scheme 1<smiles>O=C(Nc1ccccc1)Nc1ccccc1C(=O)O</smiles>

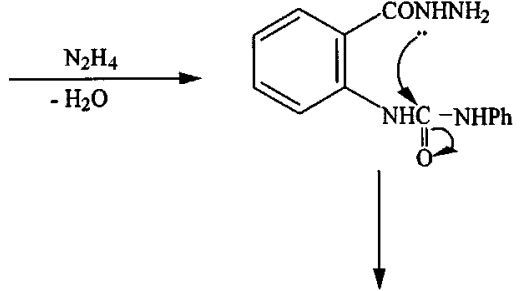<smiles>NN1C(=O)c2ccccc2NC1(O)Nc1ccccc1</smiles><smiles>C[PH+]C</smiles><smiles>N[NH+]1C(=O)c2ccccc2NC1(N)[O-]</smiles><smiles>Nn1c(O)nc2ccccc2c1=O</smiles>

Scheme 2 


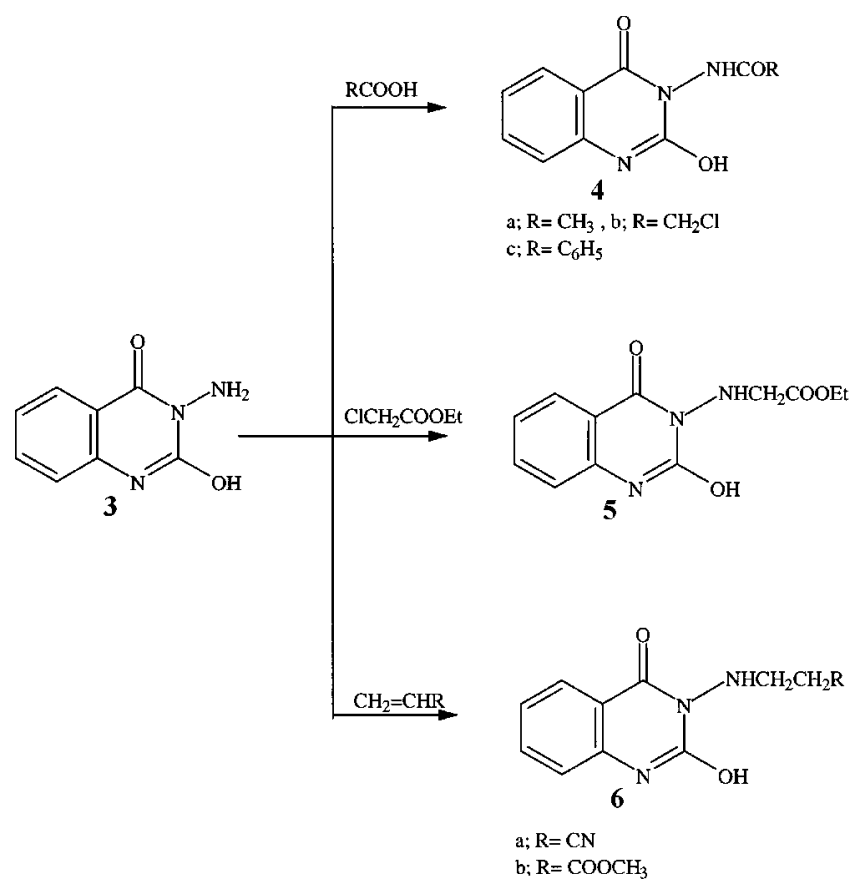

Scheme 3

Mass spectroscopy. Tables $l$ and 2 list the $m z$ (relative abundance. \%) values of the principal fragments of the studied compounds, while Figures 1,2 and 3 illustrate, as examples. the mass spectra of $3 .+\mathbf{c}$ and 6 a. respectively.

Compounds 3 and tat-c. The mass spectra (Table l) of compounds $\mathbf{3}$ and +a-c show relatively small molecular ions and peaks typical of a cleavage and rearrangement process type fragmentation. The main fragmentation pathway of compound 3 was summarized in Scheme 4 . The detection of both complementary fragments of the clearage and rearrangement processes is attributed to their comparable ionization potentials. From the study of the mass spectra of compound 3 (Figure 1), it was found that the molecular ion lad fragmented to the $m z 146$. The ion of $m z 146$ was broken to give the $m z 119$ by losing $\mathrm{HCN}$. This fragmentation led to ion of $m z 92$ and ion of $m z$ 64. respectively. However. pathway A in Table $\mathrm{l}$ is the predominant one. since the $\mathrm{mz}$ 146 which arises from the ion of $m z$ 177. is the base peak of the spectrum for all these compounds 3 and $4 \mathbf{a}$. $\mathbf{b}$.

The molecular ion of compound $+\mathbf{c}$ fragmented further and incolved two pathways as illustrated in Scheme 5 . The molecular ion of $m z 281$ fragment via the pathway $A$ to give the $m z 176$ by losing benzoyl radical group. The $m z 176$ fragmented to give the ion of $m z 1+6$ by losing hydrogen cyanide molecule to give the ion of $m z 119$.

Accordingly: the same molecular ion of $m z 281$ fragmented wa the pathway $B$ by a clearage of 3-amino-2hydroxyquinazolinone radical, has relatively low abundance (Figure 2) to give the ion of $m z 105$ which lost $\mathrm{CO}$ to give the ion of $m z 77$ (phenyl radical).

Compounds 5 and $6 \mathbf{a}, \mathbf{b}$. The mass spectra of compounds 5 and 6 a. b show relatively small molecular ions and peaks typical of a cleavage and rearrangement processes type fragmentation. From the study of the mass spectra of

Table 1. FI Mass spectra ( $70 \mathrm{eV}$ ) of compound 3 and 4 m/7. (relative intensity; \%)

\begin{tabular}{|c|c|c|c|c|c|c|}
\hline \multirow{2}{*}{ Compound } & \multirow{2}{*}{$M^{\prime}$} & \multicolumn{2}{|c|}{ Pathway A } & \multicolumn{2}{|c|}{ Pathway $B$} & \multirow{2}{*}{ Other lons: } \\
\hline & & M- & $111 z$ & M- & $\mathrm{mz}$ & \\
\hline \multirow[t]{3}{*}{3} & $\begin{array}{l}\left\lfloor\mathrm{C}_{8} \mid \mathrm{l} \mathrm{I}_{-} \mathrm{N}_{3}\left(\mathrm{O}_{2}\right\rfloor "\right. \\
177(50.21)\end{array}$ & $0-\mathrm{NII}$ & $\begin{array}{l}\left.\left[\mathrm{C}_{8} \mid \mathrm{I}_{4} \mathrm{~N}_{3} \mathrm{O}\right)\right]^{\cdot} \\
146(10(6)\end{array}$ & - & - & $\begin{array}{l}147(9.50), 120(3.06), 118(308), 105(1.47), 104 \\
(3.10), 93(3.17), 90(20.75), 77(5.30), 76(6.37), 74\end{array}$ \\
\hline & & IICN & $\begin{array}{l}{\left[\mathrm{C}-1 \mathrm{I}_{5} \mathrm{NO}\right]^{*}} \\
119(11.12)\end{array}$ & & & $\begin{array}{l}(6.15) .70)(2.72) .65(10.80) .64(20.44), 6.3(21.04) \\
62(7.53) .53(3.50) .52(10.03)\end{array}$ \\
\hline & & $11 C \mathrm{~N}$ & $\begin{array}{l}{\left[C_{n} 11,0\right]^{-1}} \\
92(27.01)\end{array}$ & & & \\
\hline \multirow[t]{3}{*}{ 4a } & $\begin{array}{l}{\left[\mathrm{C}_{10} \mathrm{H}_{2} \mathrm{~N}_{3} \mathrm{O}_{3}\right]^{-}} \\
219(2.30)\end{array}$ & $\mathrm{CH}_{2}-\mathrm{C}-\mathrm{O}$ & $\begin{array}{l}{\left[\mathrm{C}_{8} \mathrm{H}-\mathrm{N}_{3} \mathrm{O}_{2}\right]^{-}} \\
177(84.10)\end{array}$ & - & - & $\begin{array}{l}178(6.80) \cdot 147(80.60) .120(1.50) .118(1.60) .93 \\
(1.00) .92(14.30) .91(3.10) .90(1.20) .77(1.80) .\end{array}$ \\
\hline & & $\mathrm{O}^{-} \mathrm{NH}$ & $\begin{array}{c}{\left[\mathrm{C}_{8} \mathrm{H}_{\mathrm{N}} \mathrm{N}_{2} \mathrm{O}\right]^{-}} \\
146(100)\end{array}$ & & & $\begin{array}{l}76(2.7) .75(1.00) .65(4.71) .64(9.30) .63(7.6) .62 \\
(1.91) .52(3.10) .51(2.32) .50(4.70)\end{array}$ \\
\hline & & $\mathrm{HCN}$ & $\begin{array}{c}\lceil\mathrm{C} \because \mathrm{H} * \mathrm{NO}\rceil^{-} \\
119(7.20)\end{array}$ & & & \\
\hline \multirow[t]{3}{*}{$4 \mathrm{~b}$} & $\begin{array}{c}{\left[\mathrm{C}_{10} \mathrm{H}_{5} \mathrm{ClN}_{3} \mathrm{O}_{3}\right]^{--}} \\
253(2.30)\end{array}$ & $\mathrm{Cl} \mathrm{C} \cdot \mathrm{H}^{-}-\mathrm{C}^{-} \mathrm{O}$ & $\begin{array}{c}{\left[\mathrm{C}_{8} \mathrm{H}-\mathrm{N}_{3} \mathrm{O}_{2}\right]^{-}} \\
177(79.30)\end{array}$ & - & - & $\begin{array}{l}178(8.10) \cdot 148(2.80) \cdot 147(9.91) \cdot 120(1.50) .118 \\
(1.80) .104(1.20) .102(1.44) .93(1.20) .92(1600) .\end{array}$ \\
\hline & & $\mathrm{O}^{-} \mathrm{NH}$ & $\begin{array}{r}{\left[\mathrm{C}_{8} \mathrm{H}_{6} \mathrm{~N}_{2} \mathrm{O}\right]^{-}} \\
146(100)\end{array}$ & & & $\begin{array}{l}91(4.3) .90(12.00) .77(5.20) .76(3.20) .75(1.20) \\
74(1.30) .65(6.50) .64(11.20) .63(8.82) .53(1.00)\end{array}$ \\
\hline & & $\mathrm{HCN}$ & $\begin{array}{l}{\left[\mathrm{C}-\mathrm{H}_{5} \mathrm{NO}\right]^{-}} \\
119(8 . .30)\end{array}$ & & & $52(3.60) .51(5.00) .50(5.00)$ \\
\hline \multirow[t]{3}{*}{$4 c$} & $\begin{array}{l}\left\lfloor\mathrm{C}_{15} \mathrm{ll}_{11} \mathrm{~N}_{3} \mathrm{O}_{3}\right] \\
\quad 281(4 . .30)\end{array}$ & $\mathrm{Ph}-\mathrm{CO}$ & $\begin{array}{r}{\left[\mathrm{C}_{8} \mathrm{II}_{3} \mathrm{~N}_{3} \mathrm{O}_{2}\right]} \\
176(3.25)\end{array}$ & $\mathrm{C}_{8} \mathrm{H}_{6} \mathrm{~N}_{3} \mathrm{O}_{2}$ & $\begin{array}{l}{[\mathrm{Ph}-\mathrm{CO}]^{-1}} \\
105(100)\end{array}$ & $\begin{array}{l}147(1.30) .106(7.78) .91(3.55) .90(5.27) .78 \\
(4.84) .75(3.44) .74(3.05) .65(5.12) .64(8.07) .63\end{array}$ \\
\hline & & No & $\begin{array}{l}\left.\left[\mathrm{C}_{8} I \mathrm{I}_{6} \mathrm{~N}_{2} \mathrm{O}\right)\right]^{-} \\
146(8.50)\end{array}$ & $\mathrm{CO}$ & $\begin{array}{l}{\left[\mathrm{C}_{\mathrm{s}} \mathrm{II}_{\mathrm{s}}\right]^{\cdot 1}} \\
77(6 \mathrm{I} .2 .3)\end{array}$ & $(9.16) .62(2.01) .53(1.32) .52(5.12) .51(27.00)$ \\
\hline & & IICN & $\begin{array}{l}{\left[\mathrm{C}-\mathrm{II}_{\mathrm{S}} \mathrm{N}(\mathrm{)}]^{*}\right.} \\
119(12.35)\end{array}$ & & & \\
\hline
\end{tabular}


Table 2. LI Mass spectra (70 eV) ol compounds 5 and $6 \mathrm{mz} / \mathrm{L}$ (Iclative intensity: \%)

\begin{tabular}{|c|c|c|c|c|c|c|}
\hline \multirow{2}{*}{ Compound } & \multirow{2}{*}{$\mathrm{M}$} & \multicolumn{2}{|c|}{ Pathway $\Lambda$} & \multicolumn{2}{|c|}{ Pathway 13} & \multirow{2}{*}{ Other Ions } \\
\hline & & M- & $\mathrm{m} z$ & M- & $\mathrm{mz}$ & \\
\hline \multirow[t]{5}{*}{5} & $\begin{array}{c}{\left[\mathrm{C}_{12} \mathrm{H}_{1,2} \mathrm{~N}_{3} \mathrm{O}_{4}\right]^{-}} \\
263(18.30)\end{array}$ & $\mathrm{CH}_{3} \mathrm{CH}_{2} \mathrm{O}$ & $\begin{array}{l}{ }_{\mathrm{RNH}} \\
\left.\mathrm{CH}_{2} \mathrm{CO}\right]^{--} \\
218(2.60)\end{array}$ & $\mathrm{CH}_{2} \mathrm{COOEt}$ & $\begin{array}{c}{\left[\mathrm{C}_{8} \mathrm{H}_{\mathrm{n}} \mathrm{N}_{3} \mathrm{O}_{2}\right]^{+}} \\
176(3.50)\end{array}$ & $\begin{array}{l}217(7.30) .192(1.2) .191(2.20) .184(2.40) . \\
175(2.60) .133(11.00) .118(2.21) .105(7.40) . \\
103(2.00) .92(1.10) .90(3.40) .77(30.30) .78\end{array}$ \\
\hline & & $\mathrm{CO}$ & $\begin{array}{l}{\left[\mathrm{RNH} \mathrm{CH}_{2}\right]^{--}} \\
190(17.50)\end{array}$ & NO & $\begin{array}{l}{\left[\mathrm{C}_{8} \mathrm{H}_{n} \mathrm{~N}_{2} \mathrm{O}\right]^{+}} \\
146(3.60)\end{array}$ & $\begin{array}{l}(3.70) .64(1.80) .63(2.41) .51(1.3 .30) .50 \\
(5.50)\end{array}$ \\
\hline & & $\begin{array}{l}\mathrm{CH}_{2}-\mathrm{NH} \\
\quad \text { (IJ) }\end{array}$ & $\begin{array}{c}{\left[\mathrm{C}-\mathrm{H}_{4} \mathrm{~N}_{2} \mathrm{O}\right]^{+}} \\
132(1606)\end{array}$ & $\mathrm{HCN}$ & $\begin{array}{c}{\left[\mathrm{C}_{-} \mathrm{H}_{4} \mathrm{NO}^{+}\right.} \\
119(1.8)\end{array}$ & \\
\hline & & $N_{2}$ & $\begin{array}{l}\left.\left[\mathrm{C}-1 \mathrm{I}_{4} \mathrm{O}\right)\right]^{-} \\
\mathrm{l}(1) 4(5.4(1))\end{array}$ & & & \\
\hline & & co & $\begin{array}{l}{\left[C_{6} I H_{4}\right]^{\prime}} \\
76(5.70)\end{array}$ & & & \\
\hline \multirow[t]{4}{*}{$6 a$} & $\begin{array}{c}{\left[\mathrm{C}_{11} \mathrm{H}_{10} \mathrm{~N}_{1} \mathrm{O}_{2}\right]^{-}} \\
230(16.00)\end{array}$ & $\mathrm{CH}_{2}-\mathrm{CN}$ & $\begin{array}{l}{\left[\mathrm{R}-\mathrm{NH} \mathrm{CH} \mathrm{CH}_{2}\right]^{-}} \\
190(19.21)\end{array}$ & $\mathrm{CH}_{2}-\mathrm{CHCN}$ & $\begin{array}{c}{\left[\mathrm{C}_{8} \mathrm{H}=\mathrm{N}_{3} \mathrm{O}_{2}\right]^{+}} \\
177(3.84)\end{array}$ & $\begin{array}{l}178(1.20) .147(2.53) .133(9.10) .130(3.08) . \\
120(9.10) .130(3.08) .120(1.55) .117(7.55) .\end{array}$ \\
\hline & & $\begin{array}{l}\mathrm{CH}_{2}-\mathrm{NH} \\
\mathrm{CHO}\end{array}$ & $\begin{array}{l}{\left[\mathrm{C}-\mathrm{H}_{4} \mathrm{~N}_{2} \mathrm{O}\right]^{+}} \\
132(100)\end{array}$ & $\mathrm{HN}-\mathrm{O}$ & $\begin{array}{l}{\left[\mathrm{C}_{8} \mathrm{H}_{n} \mathrm{~N}_{2} \mathrm{O}\right]^{+}} \\
146(25.85)\end{array}$ & $\begin{array}{l}116(3.18) .105(11.93) .92(8.72) .91(10.20) \\
90(35.08) .77(65.42) .75(16.71) .05(10.20)\end{array}$ \\
\hline & & $\mathrm{N}_{2}$ & $\begin{array}{l}{\left[\mathrm{C}-\mathrm{H}_{+} \mathrm{O}\right\rceil^{-}} \\
](1) 4(10.55)\end{array}$ & $\mathrm{HCN}$ & $\begin{array}{l}{\left[\mathrm{C}-\mathrm{H}_{5} \mathrm{NO}^{+}\right]^{+}} \\
119(3.06)\end{array}$ & $\begin{array}{l}64(24.70), 63(27.50), 62(11.36) .58(30.1 .3) . \\
54(25.51), 5.3(10.34), 52(3.40), 51(40.3(1)\end{array}$ \\
\hline & & CO & $\begin{array}{l}\left\lfloor\mathrm{C}_{\mathrm{t}}, \mathrm{II}_{4}\right\rfloor^{-1} \\
76(24.72)\end{array}$ & - & - & \\
\hline \multirow[t]{4}{*}{$6 b$} & $\begin{array}{c}{\left[\mathrm{C}_{12} \mathrm{H}_{1,3} \mathrm{~N}_{3} \mathrm{O}_{1}\right]^{-}} \\
263(24,05)\end{array}$ & $\mathrm{CH}_{3} \mathrm{O}$ & $\begin{array}{l}{\left[\mathrm{RNHC} \mathrm{H}_{2-}^{-}\right.} \\
\left.\mathrm{CH}_{2} \mathrm{CO}\right\rceil^{-} \\
232(5.78)\end{array}$ & $\begin{array}{c}\mathrm{CH}_{2} \mathrm{CHCOO} \\
\mathrm{Mc}\end{array}$ & $\begin{array}{c}{\left[\mathrm{C}_{8} \mathrm{H}-\mathrm{M}_{3} \mathrm{O}_{2}\right]^{-}} \\
177(4.83)\end{array}$ & $\begin{array}{l}23.1(8.30) .192(5.35) .178(2.21) .147(3.05) \\
133(10.05) .130(4.02) .120(1.62) .117(11.25) \\
116(6.21) .105(16.13) .62(14.23) .91(15.37)\end{array}$ \\
\hline & & $\mathrm{CH}_{2}-\mathrm{C}-\mathrm{O}$ & $\begin{array}{l}\left\lceil\mathrm{RNHCH}_{2}\right\rceil^{+} \\
190(23.50)\end{array}$ & $\mathrm{HN}-\mathrm{O}$ & $\begin{array}{l}{\left[\mathrm{C}_{8} \mathrm{H}_{n} \mathrm{~N}_{2} \mathrm{O}\right]^{+}} \\
146(21.38)\end{array}$ & $\begin{array}{l}90(43.12) .77(62.71) .75(16.20) .65(13.20) . \\
64(27.21) .6 .3(32.01) .62(13.26)\end{array}$ \\
\hline & & $\begin{array}{l}\mathrm{CH}_{2}-\mathrm{NH} \\
\quad \mathrm{CII}\end{array}$ & $\begin{array}{c}{\left[\mathrm{C}_{-}-\mathrm{H}_{4} \mathrm{~N}_{2} \mathrm{O}\right]^{+}} \\
1.32(1)(6)\end{array}$ & $\mathrm{HCN}$ & $\begin{array}{l}{\left[\mathrm{C}_{-} \mathrm{H}_{3} \mathrm{NO}^{+}\right.} \\
1 \mathrm{II}(7 . .31)\end{array}$ & \\
\hline & & $\mathrm{N}_{2}$ & $\begin{array}{l}{\left[\mathrm{C}-1 \mathrm{I}_{+}(\mathrm{O}]^{-}\right.} \\
\mathrm{I}(1) 4(9 . .35)\end{array}$ & - & - & \\
\hline
\end{tabular}

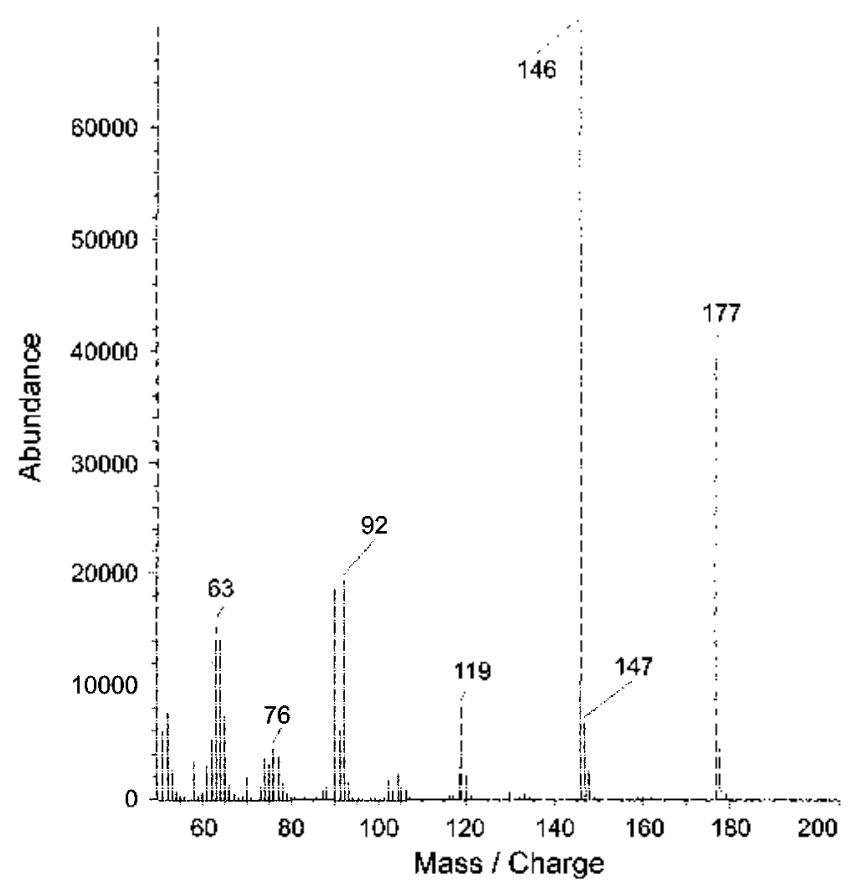

Figure 1.70 eV mass spectrum of 3

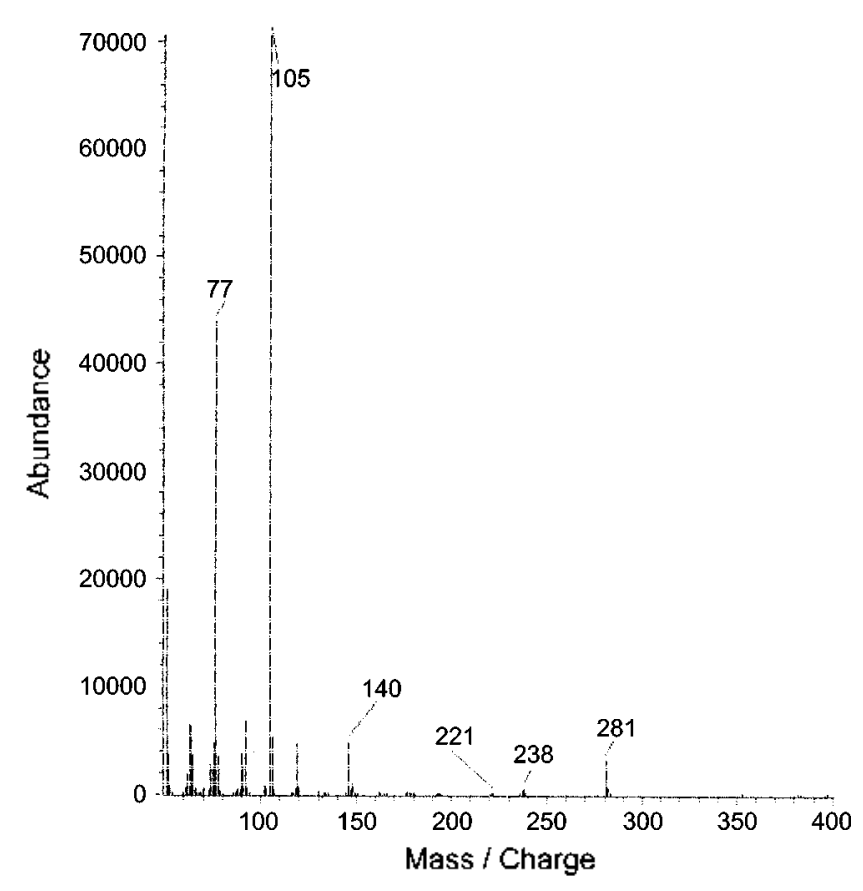

Figure 2. $70 \mathrm{cV}$ mass spectrum of $4 \mathrm{c}$. 


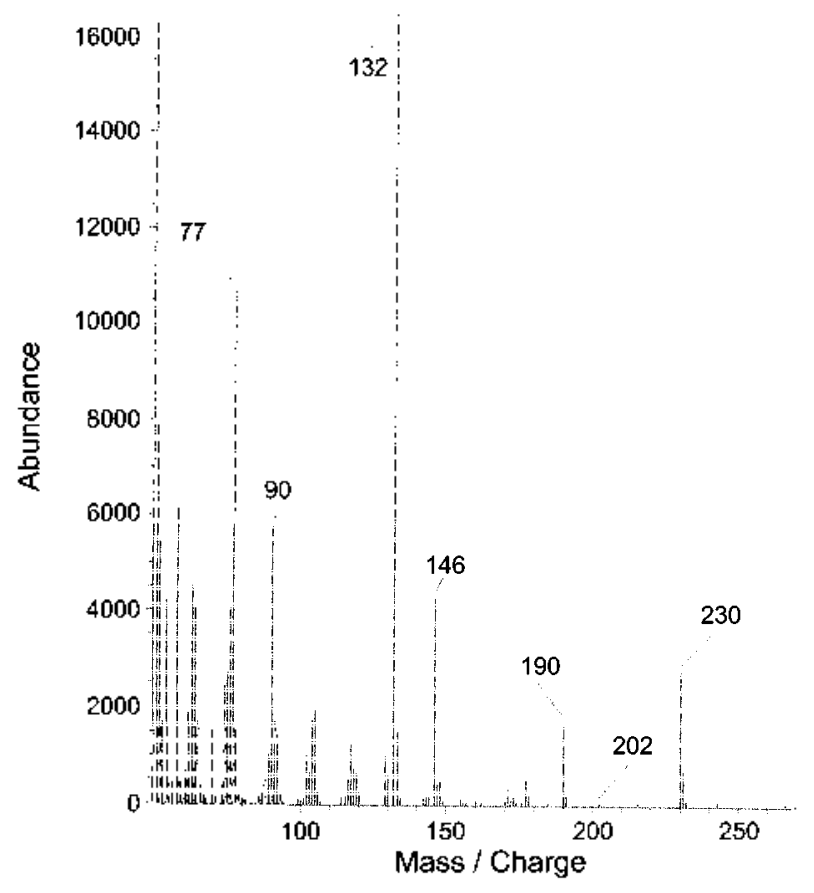

Figure 3. $70 \mathrm{eV}$ mass spectrum of 6a.

compounds 5 and $6 \mathbf{a}$. $\mathbf{b}$. it was found that the molecular ion for all these compounds fragmented further and involved two various pathways as illustrated by Scheme 6 as representatice examples.

The main fragmentation pathways of compounds 5 . and 6a. b are summarized in Table 2. However, the molecular ion of $m z 230$ fragmented via the pathway A to gave the ion of $m z 190$. which fragmented further to give the stable ion of $m z 132$ by losing $\mathrm{CH}_{2}=\mathrm{NH}$ and fromyl radical group.

Subsequently, the molecular ion of $m z 230$ was broken via pathway $B$ in the same fragmentation processes which was observed for compounds 3, and 4a. c (Pathway A).

\section{Experimental Section}

Melting points were determined on a Boetium Hostage apparatus and uncorrected. IR spectra were recorded on a Perkin-Elmer FTIR 1725 spectrometer. The 'H-NMR spectra were recorded on a General Electric QE 300. and chemical shifts were given with respect to TMS. Mass spectra were recorded on a VG Autspec CEI FAB' and a Hew lett Packard Ms-Engine thermospray and ionization by electron impact at $70 \mathrm{eV}$. The accelerating voltage was $6 \mathrm{kV}$, the temperature of the ion source was $\sim 200^{\circ} \mathrm{C}$ and the emission current was $\sim 100 \mathrm{~mA}$. Microanalyses were conducted using an elemental analyzer 116.

3-Amino-2-hyddroxy-4 (3H)-quinazolinone (3). A mixture of $1(0.01 \mathrm{~mol})$ and hydrazine hydrate $(0.03 \mathrm{~mol})$ was fused on a hot plate for $10-15 \mathrm{~min}$. the reaction mixture was added to boiling methanol $(50 \mathrm{~mL})$ and heated under reflux for $2 \mathrm{~h}$. then cooled. The solid formed was filtered off, washed with methanol. dried and purified by recrystallization with dimethyl formanide to give 3 as colourless crystals. yield
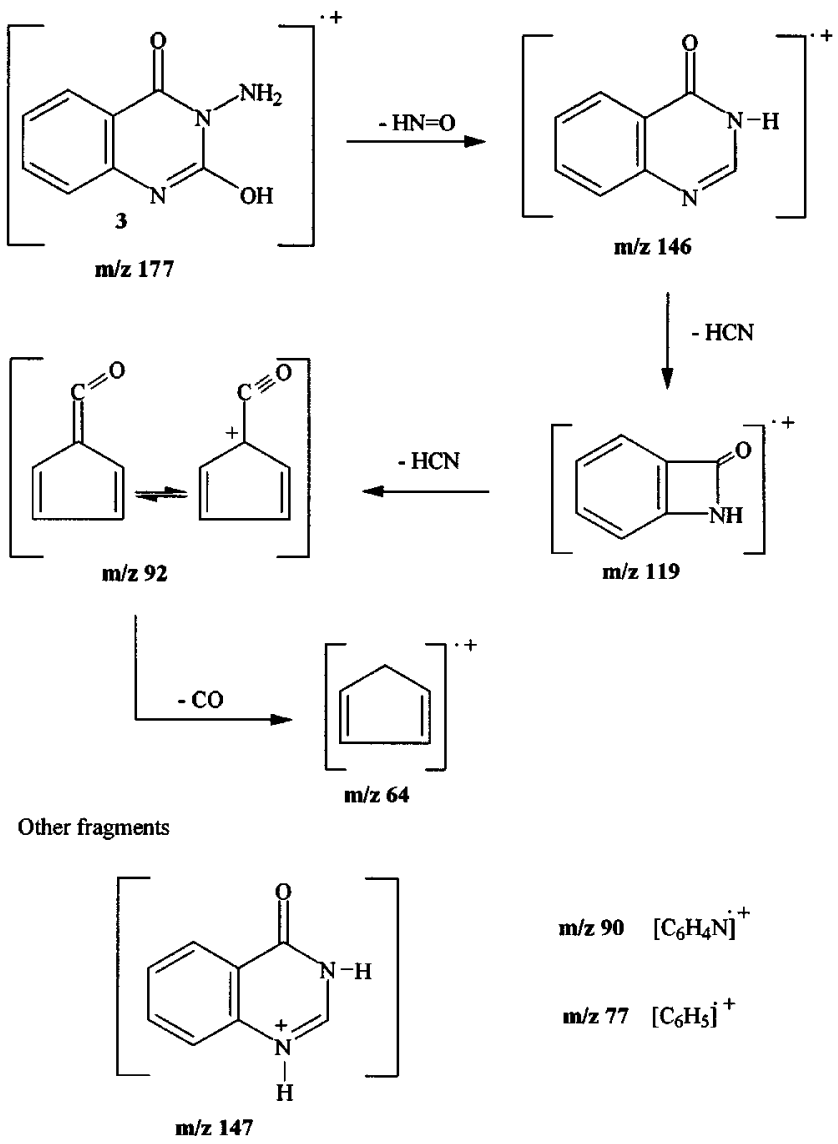

Scheme 4. Main fragmentation palhway of compound 3.

73\%. mp 275. IR (KBr) 3320. $3215\left(\mathrm{NH}_{2}\right) .3390-2850$ (br. $\mathrm{OH}), 1689(\mathrm{CO}) .1625(\mathrm{C}=\mathrm{N}) \mathrm{cm}^{-1}:{ }^{1} \mathrm{H}-\mathrm{NMR}\left(\mathrm{DMSO}-\mathrm{d}_{6}\right) \delta$ 5.48 (s. $2 \mathrm{H} . \mathrm{NH}_{2}$ ) $7.22-7.95$ (m. $4 \mathrm{H}$. ArH) ppm: Found: $\mathrm{C}$. 54.03: H. 3.62: N. 23.57. $\mathrm{C}_{8} \mathrm{H}-\mathrm{N}_{3} \mathrm{O}=$ requires: $\mathrm{C}, 54.24: \mathrm{H}$. $3.95:$ N. 23.73.

3-(Alkylcarbonyl)amino-2-hydroxy- $4(3 \mathrm{H})$-quinazolinones (4a-c). A mixture of $3(0.0 \mathrm{~L} \mathrm{~mol})$ and appropriate acids such as acetic acid. chloroacetic acid. and benzoic acid $(0.01 \mathrm{~mol})$ in phosphours oxychloride $(30 \mathrm{~mL})$ was heated on a water bath under reflux for $30 \mathrm{~min}$. then cooled and poured onto ice-water. The resulting product was filtered. washed with water. dried and purified by recrystallization with ethanol to give 4.

3-(Acetyliamino-2-hydroxy-4(3H)-quinzaolinone (ta): yield $63 \%$. mp 220. IR (KBr) $3225(\mathrm{NH}) .3389-2850$ (br. OH) 1695-1686 $(\mathrm{C}=\mathrm{O}) .1623(\mathrm{C}=\mathrm{N}) \mathrm{cm}^{-1}:$ ' H-NMR (DMSOd $\left.\mathrm{d}_{6}\right) \delta 2.01\left(\mathrm{~s}, 3 \mathrm{H}, \mathrm{CH}_{3}\right), 7.2 \mathrm{l}-7.96(\mathrm{~m},+\mathrm{H}, \mathrm{ArH}), \mathrm{10} .49$ (s. 1H. NH) ppm: Found: C. 54.54: H. 4.02: N. 19.06 . $\mathrm{C}_{1}\left(\mathrm{H}_{i} \mathrm{~N}_{3} \mathrm{O}_{3}\right.$ requires: C. 54.79: $\mathrm{H}, 4.11: \mathrm{N} .19 .18$.

3-(Chloroacetyllamino-2-hvdroxy-4 (3H)-quinazolinone $(t h)$ : yield $6+\% . \mathrm{mp} 205^{\circ}$. IR( $\left.\mathrm{KBr}\right) 3229(\mathrm{NH}) .3396-$ 2851 (br. $\mathrm{OH}) .1699-1682(\mathrm{C}=\mathrm{O}) .1625(\mathrm{C}=\mathrm{N}) \mathrm{cm}^{-1}$ : $\mathrm{H}-$ NMR (DMSO-d d $_{6} \delta 5.03$ (s. 2H. $\left.\mathrm{CH}_{2}\right) .7 .21-7.95(\mathrm{~m} .+\mathrm{H}$. ArH) 10.50 (s. IH. NH) ppm: Found: C. 47.11: H. 3.01: N. 16.32. $\mathrm{Cl}$. 13.68. $\mathrm{C}_{1} \mathrm{H}_{8} \mathrm{ClN}_{3} \mathrm{O}_{3}$ requires: C. 47.34: H. 3.16: N. $16.57: \mathrm{Cl}$. $1+.00$.

3-(Benzovllamino-2-hydoxy-4(3H)-quinazolinone (4c): 


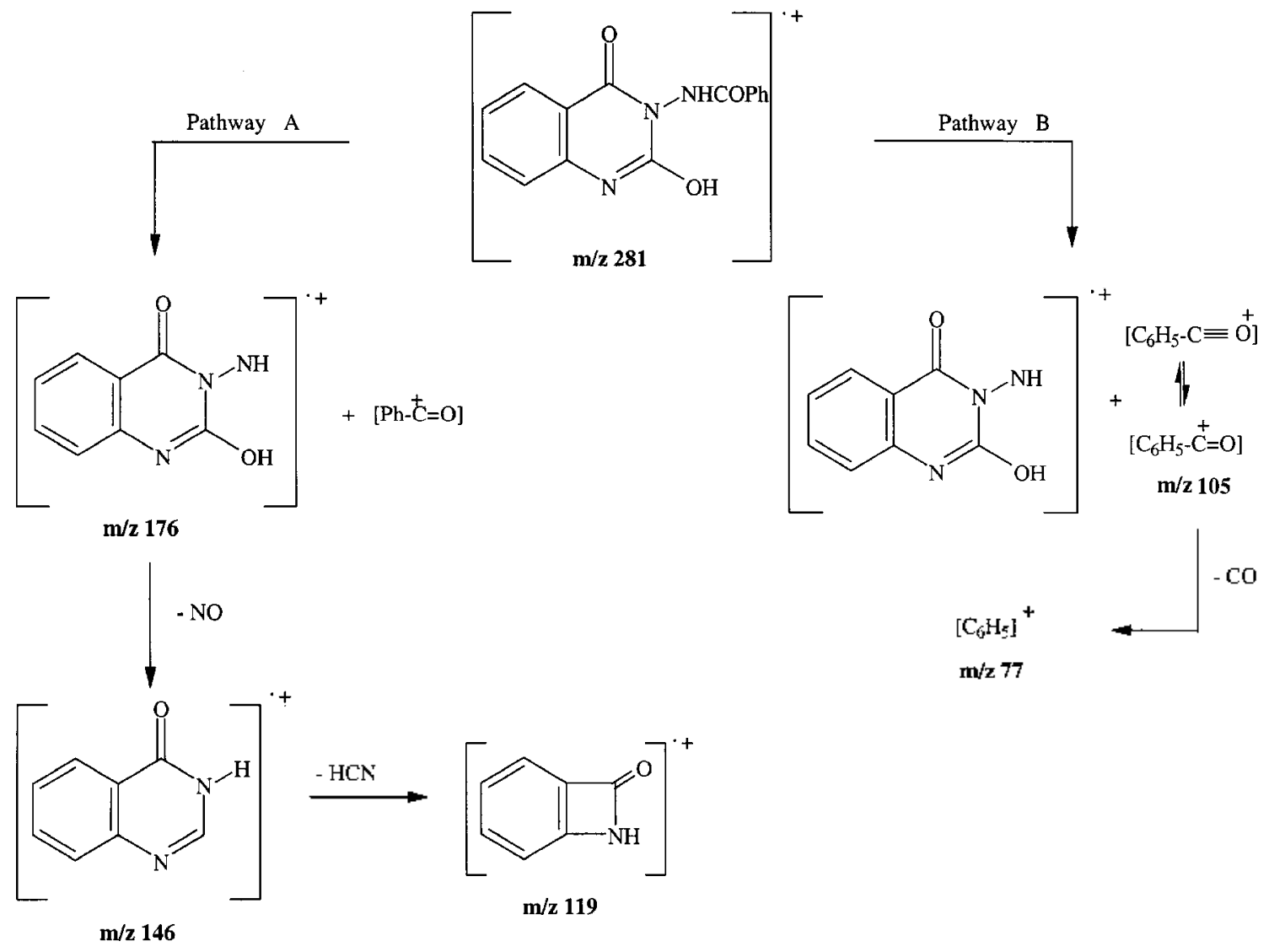

Other fragments

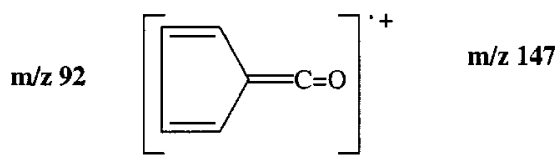<smiles>CCCCCC</smiles>

\author{
$\mathbf{m} / \mathbf{2} \mathbf{1 0 6}\left[\mathrm{C}_{6} \mathrm{H}_{5} \mathrm{CHO}\right]^{+}$ \\ $\mathrm{m} / \mathbf{z} 78\left[\mathrm{C}_{6} \mathrm{H}_{5}\right]^{+}+$ \\ $\mathbf{m} / \mathbf{z} 90\left[\mathrm{C}_{6} \mathrm{H}_{4} \mathrm{~N}\right]^{\prime+}$
}

Scheme 5. Main fragmentation pathway of compound tc

yield 67\%. Imp 227\%, IR (KBr) $3215(\mathrm{NH}) .33+5-282 \mathrm{l}$ (br. OH). $1695-1681(\mathrm{C}=\mathrm{O}) .162+(\mathrm{C}=\mathrm{N}) \mathrm{cm}^{-1}: \mathrm{H}-\mathrm{NMR}$ (DMSO$\left.\mathrm{d}_{6}\right) \delta 7.2 \mathrm{l}-8.01(\mathrm{~m}, 9 \mathrm{H}, \mathrm{ArH}) .10 .6 \mathrm{l}(\mathrm{s}, \mathrm{H} . \mathrm{NH}) \mathrm{ppm}$; Found: C. $63.89 ; \mathrm{H}, 3.66 ; \mathrm{N} .14 .59 \cdot \mathrm{C}_{1}=\mathrm{H}_{11} \mathrm{~N}_{3} \mathrm{O}_{3}$ requires: $\mathrm{C}$. $64.06:$ H. 3.91: N. 14.95 .

3-(Alkyl)amino-2-hydroxy-4(3H)-quinazolinones 5 and 6. A mixture of $3(0.01 \mathrm{~mol})$ and a reagent such as ethyl cluloroacetate, acrylonitrile and methyl acrylate (0.01 mol) in dimethyl formamide $(30 \mathrm{~mL})$ was heated under relux for th. then cooled and poured into water. the deposited solid was filtered off, washed with water. dried and purified by recrystallization with ethanol to give 5 an 6 . respectively:

Fihy 2-hvaroxv-t(3H)-quinazolin-3-vhinoacetic ester $(5)$. yield 67\%. mp 135\%: IR (KBr) $3225(\mathrm{NH}) .3396-2852$ (br. $\mathrm{OH}) .1765\left(\mathrm{C}=\mathrm{O}\right.$ of ester). $1686(\mathrm{CO}) .1622(\mathrm{C}=\mathrm{N}) \mathrm{cm}^{-1},{ }^{1} \mathrm{H}-$ NMR (DMSO-d 6 ) $\delta 1.30\left(\mathrm{t}, J=9.04 \mathrm{~Hz} .3 \mathrm{H}, \mathrm{CH}_{3}\right) .3 .52$ (s. $\left.2 \mathrm{H}, \mathrm{CH}_{2}\right), 4.35\left(\mathrm{q}, J=6.23 \mathrm{~Hz}, 2 \mathrm{H}, \mathrm{OCH}_{2}\right) .5 .81$ (s. $\mathrm{lH}$.
$\left.\mathrm{NHCH}_{2}\right), 7.21-7.96(\mathrm{ml}, 4 \mathrm{H}, \mathrm{ArH})$ ppm: Found: C. 54.52. H, $4.69 ; \mathrm{N}, 15.59 \cdot \mathrm{C}_{1} \mathrm{H}_{1.3} \mathrm{~N}_{3} \mathrm{O}_{4}$ requires: C. $54.75 . \mathrm{H} .4 .94: \mathrm{N}$. 15.97 .

3-(c) vanoethyllamino-2-hwdroxy-4(3H)-quinazolinone (6a): yield $67 \%$. mp 185. IR (KBr) $3225(\mathrm{NH})$. 3389-285l br. OH). $2225(\mathrm{C}=\mathrm{N}) .1689(\mathrm{CO}) .1626(\mathrm{C}=\mathrm{N}) \mathrm{cm}^{-1}:{ }^{1} \mathrm{H}-\mathrm{NMR}$ (DMSO-d 6 ) $82.93-2.97\left(\mathrm{t} . J=9.04 \mathrm{~Hz}, 2 \mathrm{H} . \mathrm{NHCH}_{2}\right) .4 .43-$ +.47 (t. $J=9.12 \mathrm{H}_{2} .2 \mathrm{H} . \mathrm{CH}_{2} \mathrm{CN}$ ). $5.6 \mathrm{l}$ (s. $1 \mathrm{H} . \mathrm{NH}$ ). $7.2 \mathrm{l}-$ 7.96 (m. +H. ArH) ppm: Found: C. 57.02: H. +.12: N. 24.10. $\mathrm{C}_{11} \mathrm{H}_{1}, \mathrm{~N}_{4} \mathrm{O}_{2}$ requires: C. 57.39: H. 4.35: N. 24.35 .

Methy 2-hvdroxy-4 (3H)-oxo-quinazolin-3-vlaminopropionic ester $(6 h)$ : yield $65 \%$. mp 145\%. IR (KBr) 3229 (NH), 3396-285l (br. OH). 1766 (CO of ester). 1687 (CO). $162+(\mathrm{C}=\mathrm{N}) \mathrm{cm}^{-1}$ : ${ }^{1} \mathrm{H}-\mathrm{NMR}\left(\mathrm{DMSO}_{\mathrm{d}}\right) \delta 2.53-2.56(\mathrm{t} . J=$ $\left.9.0+\mathrm{Hz}, 2 \mathrm{H} . \quad \mathrm{COCH}_{2}\right), 2.92-2.97(\mathrm{t}, J=9.12 \mathrm{~Hz}, 2 \mathrm{H}$. $\left.\mathrm{NHCH}_{2}\right), 3.93\left(\mathrm{~s}, 3 \mathrm{H}, \mathrm{OCH}_{3}\right), 5.62(\mathrm{~s}, \mathrm{lH}, \mathrm{NH}), 7.22-7.96$ (m. 4H, ArH) ppm; Found: C. 54.51: H. 4.73: N. 15.72. $\mathrm{C}_{1} \mathrm{H}_{1.3} \mathrm{~N}_{3} \mathrm{O}_{4}$ requires: C. $54.75 ; \mathrm{H} .4 .94, \mathrm{~N} .15 .97$. 


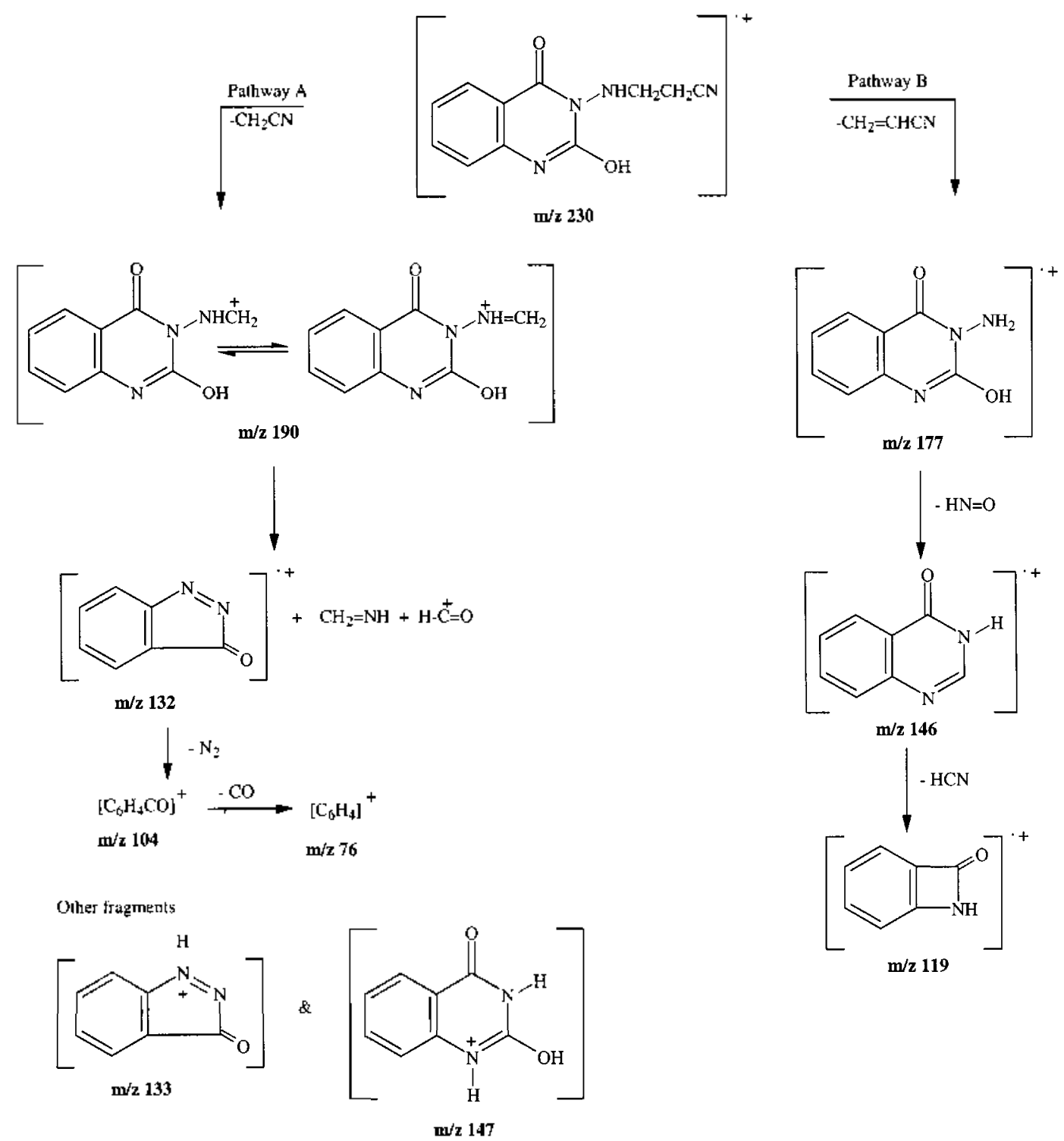

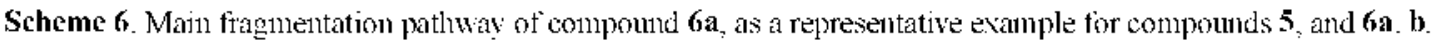

\section{References}

1. El-Deen. 1. M. J. Serb. Chem. Soc. 1998.93(12).915.

2. l'apudopoulos. E. l': Torres. C. D. J. Hetenechefic Chem. 1982. 19. 269 .

3. FI-Desulk S. Fi-Deen. J. M. J. Sewh (Them Soc. 1992, $57,513$.

4. FI-Deen, I. M.: Mohamed, S. M. Indian J. Heterowed (Them. 1993, 2, 233 (Chem. Abstr. 1994, 120, 298578).
5. Fl-Deen, I. M.: Mohamed, S. M.: Ismail. M. M.: Abdel-Megid, M.An. Onim. 1993. 89.621 (Chem. Abstr. 1994. 120. 323471).

6. Mohamed. E. A.: El-Deen. I. M.: Ismail. M. M. I'ak. J. Ind Res. 1992. 2. 1427 (Chem. Abstr. 1993. 119.8778).

7. Molamed, Г.. A.: Г.।-Deen. I. M.: Ismail, M. M.: Moltancd, S. M Ihdian .J. (he'm. 1993, 32. 933 (Chem. Abstr. 1994, 120. 244927).

8. Abd F.1-Fattah, M. F. Indian .J. Heternckel. ('he'm. 1995, f. 199 (Chem. Abstr. 1995. 132.83306). 